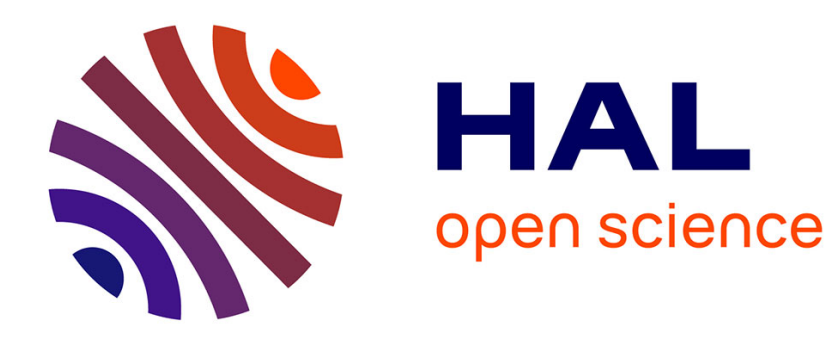

\title{
Contamination d'un plasma d'argon par des vapeurs anodiques de cuivre
}

\author{
P. Andanson, B. Cheminat
}

\section{To cite this version:}

P. Andanson, B. Cheminat. Contamination d'un plasma d'argon par des vapeurs anodiques de cuivre. Revue de Physique Appliquée, 1979, 14 (8), pp.775-782. 10.1051/rphysap:01979001408077500 . jpa00244659

\section{HAL Id: jpa-00244659 https://hal.science/jpa-00244659}

Submitted on 1 Jan 1979

HAL is a multi-disciplinary open access archive for the deposit and dissemination of scientific research documents, whether they are published or not. The documents may come from teaching and research institutions in France or abroad, or from public or private research centers.
L'archive ouverte pluridisciplinaire HAL, est destinée au dépôt et à la diffusion de documents scientifiques de niveau recherche, publiés ou non, émanant des établissements d'enseignement et de recherche français ou étrangers, des laboratoires publics ou privés. 


\title{
Contamination d'un plasma d'argon par des vapeurs anodiques de cuivre
}

\author{
P. Andanson et B. Cheminat \\ Laboratoire de Physique des Matériaux, Université de Clermont-Ferrand, B.P. 45, F 63170 Aubière, France \\ (Reçu le 31 janvier 1979, révisé le 17 avril 1979, accepté le 7 mai 1979)
}

\begin{abstract}
Résumé. - La région proche de l'anode en cuivre $(1 \mathrm{~mm}$ à $1 \mathrm{~cm})$ d'un arc stabilisé sous atmosphère d'argon $(15 \mathrm{~A}$ et $30 \mathrm{~A})$ est étudiée. Un abaissement de température important dû à la présence des vapeurs métalliques ainsi que la répartition concentrique et périphérique du cuivre sont mis en évidence. La concentration en cuivre se situe entre $10^{-2}$ et $10^{-5}$. La méthode permettant de calculer les différents paramètres à partir des valeurs expérimentales est indiquée.
\end{abstract}

\begin{abstract}
The region close to the copper anode $(1 \mathrm{~mm}$ to $1 \mathrm{~cm})$ of a stabilized arc discharge in argon (15 A and $30 \mathrm{~A}$ ) is studied. An important drop in temperature due to the metal vapor as well as the concentric and peripherical copper distribution are shown up. The copper concentration is between $10^{-2}$ and $10^{-5}$. The method allowing to compute the different parameters is given.
\end{abstract}

\section{Liste des symboles}

$\varepsilon(r) \quad$ coefficient d'émissivité locale d'une raie

$T \quad$ température

$N_{\mathrm{e}} \quad$ densité électronique

$X \quad$ teneur en cuivre

$N_{\mathrm{Ar}} \quad$ densité d'atome d'argon

$N_{\mathrm{Ar}^{+}}$densité d'ion argon

$N_{\mathrm{Cu}}$ densité d'atome de cuivre

$N_{\mathrm{Cu}^{+}}$densité d'ion cuivre

$U_{Z} \quad$ fonction de partition de la particule $Z$

\section{$p \quad$ pression}

$k$

$m_{\mathrm{e}}$

$h$

$E_{\alpha_{\mathbf{A r}}}$

$E_{\alpha_{\mathrm{Cu}}}$

$N_{m}$

$g_{m} \quad$ poids statistique du niveau $m$

$E_{m} \quad$ énergie du niveau $m$ de l'atome

$d$ distance de l'anode à la section de visée

rayon (dans la géométrie cylindrique)

Nous présentons une étude expérimentale sur la contamination d'un plasma d'arc par des vapeurs métalliques de cuivre, l'arc brûlant dans de l'argon.
Des travaux ont été faits récemment dans l'air [1-6] et dans l'azote [1] pour des intensités variant de 1 à $15 \mathrm{~A}$. On trouve également une étude à forte intensité $\mathrm{W} / \mathrm{cm}^{3}$.str. dans le $\mathrm{SF}_{6}$ [6]. Les vapeurs métalliques sont géné$\mathrm{K}$ ralement des vapeurs de cuivre qui est un métal très $\mathrm{cm}^{-3} \quad$ utilisé dans les contacts électriques et qui a des pro$\mathrm{cm}^{-3} \quad$ part des autres métaux.

Nous avons constaté, à la suite d'études sur un plasma d'argon dans une chambre de Maecker que, dans le cas d'une anode en cuivre, un important dépôt de ce métal se fait sur le cône servant de guide au pied d'arc. Les vapeurs métalliques étant localisées près de l'anode, nous avons construit une chambre spéciale permettant des mesures au voisinage de cette électrode (entre $1 \mathrm{~mm}$ et $1 \mathrm{~cm}$ ). Le diagnostic spectroscopique de ce plasma argon-cuivre nécessite un traitement mathématique permettant à partir de l'intensité des raies spectrales de déduire les valeurs locales des densités des différentes particules ainsi que la température et la teneur en cuivre. Les mesures nécessitent une émission de vapeurs métalliques continue et relativement stable dans le temps (plusieurs minutes). C'est un problème difficile, nous indiquerons les conditions expérimentales et les principaux paramètres influant sur la stabilité. Les résultats expérimentaux exposés ont été confirmés par de très nombreux essais afin d'éliminer au maximum les erreurs dues à une variation du taux de cuivre dans le plasma.

1. Calcul des paramètres du plasma à partir des intensités totales de raies. - Dans le cas d'un arc à 
symétrie cylindrique, on obtient expérimentalement la répartition radiale de l'intensité d'une raie. L'inversion de l'équation intégrale d'Abel [7] permet d'obtenir la valeur du coefficient d'émissivité locale. Un étalonnage, à l'aide d'une lampe à filament de tungstène, permet de déduire la valeur absolue du coefficient d'émissivité locale $\varepsilon(r)$ pour chaque valeur de $r$. Cette détermination est faite pour plusieurs raies et permet le calcul des différents paramètres caractérisant le plasma argon-cuivre étudié.

Le calcul de la composition d'équilibre d'un plasma argon-cuivre pour des températures comprises entre $5000 \mathrm{~K}$ et $14000 \mathrm{~K}$ est donné dans [8]. Nous avons considéré comme négligeables les densités d'ions $\mathrm{Ar}^{++}$ et $\mathrm{Cu}^{++}$. Les valeurs élevées des potentiels d'ionisation des ions $\mathrm{Ar}^{+}$et $\mathrm{Cu}^{+}$(respectivement 27,62 et 20,29 eV) ainsi que la limite supérieure du domaine de température considéré justifient cette hypothèse.

Nous rappelons brièvement le système d'équations utilisé :

Equation des gaz parfaits :

$$
p=k T\left(N_{\mathrm{e}}+N_{\mathrm{Ar}}+N_{\mathrm{Ar}^{+}}+N_{\mathrm{Cu}}+N_{\mathrm{Cu}^{+}}\right) \text {. }
$$

Equation de neutralité électrique :

$$
N_{\mathrm{e}}=N_{\mathrm{Ar}^{+}}+N_{\mathrm{Cu}^{+}} \text {. }
$$

Définition de la teneur en cuivre :

$$
X=\frac{N_{\mathrm{Cu}}+N_{\mathrm{Cu}^{+}}}{N_{\mathrm{Ar}}+N_{\mathrm{Ar}^{+}}} .
$$

Equation de Saha appliquée au cuivre et à l'argon :

$$
\begin{aligned}
& \frac{N_{\mathrm{e}} N_{\mathrm{Ar}^{+}}}{N_{\mathrm{Ar}}}=\frac{\left(2 \pi m_{\mathrm{e}} k T\right)^{3 / 2}}{h^{3}} \frac{2 U_{\mathrm{Ar}^{+}}}{U_{\mathrm{Ar}}} \times \\
& \quad \times \exp \left(-\frac{1}{k T}\left(E_{\alpha \mathrm{Ar}}-\Delta E\right)\right)=S 1 \\
& \begin{aligned}
\frac{N_{\mathrm{e}} N_{\mathrm{Cu}^{+}}}{N_{\mathrm{Cu}}}= & \frac{\left(2 \pi m_{\mathrm{e}} k T\right)^{3 / 2}}{h^{3}} \frac{2 U_{\mathrm{Cu}^{+}}}{U_{\mathrm{Cu}}} \times \\
& \times \exp \left(-\frac{1}{k T}\left(E_{\alpha \mathrm{Cu}}-\Delta E\right)\right)=S 2
\end{aligned}
\end{aligned}
$$

$\Delta E$ représente l'abaissement du potentiel d'ionisation. Nous avons pris pour cette valeur l'expression de Debye-Hückel [9].

Ce système d'équation peut être complété par l'équation de Boltzmann appliquée aux niveaux $m 1$ de l'argon et $m 2$ du cuivre.

$$
\begin{aligned}
& N_{m 1}=\frac{N_{\mathrm{Ar}} g_{m 1}}{U_{\mathrm{Ar}}} \exp \left(-\frac{E_{m 1}}{k T}\right) \\
& N_{m 2}=\frac{N_{\mathrm{Cu}} g_{m 2}}{U_{\mathrm{Cu}}} \exp \left(-\frac{E_{m 2}}{k T}\right) .
\end{aligned}
$$

Les fonctions de partition des différentes espèces de particules sont tabulées dans [10].
La mesure de l'intensité absolue de certaines raies permet à partir de la relation :

$$
\varepsilon(r)=\frac{1}{4 \pi} A_{m n} N_{m} h v_{0}
$$

de déterminer $N_{m 1}$ et $N_{m 2}$.

(1) et (2) permettent d'écrire :

$$
2 N_{\mathrm{e}}+N_{\mathrm{Ar}}+N_{\mathrm{Cu}}-\frac{p}{k T}=0
$$

(4) et (5) combinées à (2) donnent l'équation suivante :

$$
S 2 N_{\mathrm{Cu}}+S 1 N_{\mathrm{Ar}}-N_{\mathrm{e}}^{2}=0 .
$$

Pour un couple de valeur $\left(T, N_{\mathrm{e}}\right)$ donné a priori $N_{\text {Ar }}$ et $N_{\text {Cu }}$ sont déterminés. Il suffit alors que $T$ et $N_{\mathrm{e}}$ soient solutions de (8) et (9). La recherche des racines se fait par projections orthogonales. $N_{\mathrm{e}}$ et $T$ sont calculées par dichotomie.

Pratiquement nous avons pris comme domaine d'étude de température les limites de validité du système d'équations [8] : $5000 \mathrm{~K}<T<14000 \mathrm{~K}$ et pour la densité électronique :

$$
10^{10}<N_{\mathrm{e}}<10^{20} \text { électrons } / \mathrm{cm}^{3} .
$$

Nous avons testé le programme de calcul en utilisant les résultats de [8]. Le temps de calcul est de l'ordre de 3 s sur un PDP 10 Digital Equipement si on impose une précision de $10^{-6}$ sur $N_{\mathrm{e}}$ et de $10^{-4}$ sur $T$.

Le calcul effectué sur plusieurs couples de raies (Ar-Cu) permet d'obtenir plusieurs valeurs des différents paramètres. La détermination de la température par la méthode des intensités relatives de raies permet une comparaison supplémentaire.

2. Dispositif expérimental. - Le dispositif de mesures spectrométriques (Fig. 1), piloté par microprocesseur, est celui décrit dans [11]; il est complété par la commande par le microprocesseur du déplacement latéral de la chambre à arc, qui se fait par l'intermédiaire d'un moteur pas à pas. Nous avons ajouté à ce montage un système optique permettant de projeter l'image de la colonne sur un écran (avec grossissement de l'ordre de 10) et de surveiller ainsi l'émission de vapeurs métalliques. Le temps de mesure est de l'ordre de quelques minutes pour une étude faite sur 6 raies (exploration radiale de 6 raies et des 6 fonds continus correspondants au pas de $0,5 \mathrm{~mm}$ ). Ce temps peut difficilement être réduit car il dépend essentiellement du temps nécessaire au positionnement du réseau.

Nous avons utilisé comme chambre à arc une chambre ayant un seul anneau de refroidissement, assez courte $(5 \mathrm{~cm})$, avec une ouverture latérale sur toute la hauteur de la chambre afin de permettre des mesures dans n'importe quelle zone du plasma. L'arrivée d'argon se fait tangentiellement de manière à ne pas perturber la colonne. 


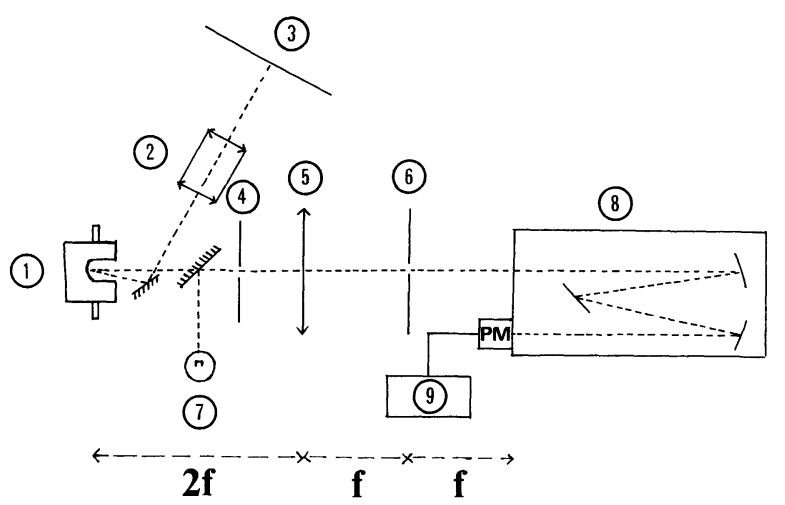

Fig. 1. - Schéma du dispositif expérimental : 1 - chambre à arc au niveau de la visée, 2 - système optique de projection, 3 - écran, 4 - fente horizontale $(0,3 \mathrm{~mm}), 5$ - lentille de distance focale $f$, 6 - trou calibré $(200 \mu \mathrm{m}), 7$ - lampe étalon, 8 - monochromateur, 9 - acquisition de données.

[Diagram of the experimental device : 1 - arc chamber at the viewing plane, 2 - projection optical system, 3 - screen, 4 - horizontal split $(0.3 \mathrm{~mm}), 5$ - lens with a $f$ focal length, 6 - calibrated hole $(200 \mu \mathrm{m}), 7$ - standard lamp, 8 - monochromator, 9 - data aquisition.]

La stabilité de l'émission de vapeurs de cuivre dans la colonne dépend d'un certain nombre de facteurs dont les plus importants sont :

- le refroidissement de l'anode,

- le débit d'argon,

- la nature et la forme des électrodes.

Nous avons travaillé dans les conditions suivantes :

Cathode : elle est en tungstène, elle a la forme d'un cylindre $(\varnothing 6 \mathrm{~mm})$ taillé en pointe (angle de $\left.30^{\circ}\right)$. La pointe favorise la stabilité du spot cathodique qui a une influence importante sur la stabilité de la colonne.

Anode : elle est en cuivre (pureté électrolytique) ou en cuivre contenant du zirconium $(0,1 \%)$, cylindrique $(\varnothing 6 \mathrm{~mm})$ et plate. La présence de zirconium permet une usure moindre mais ne semble pas favoriser la stabilité.

Argon : le débit est de 1,7 1/min. Si le débit d'argon est trop faible $(<0,4 \mathrm{l} / \mathrm{min})$ l'air pénètre dans la chambre, il y a une très intense émission de cuivre et l'arc change de régime. Au-dessous de $0,3 \mathrm{l} / \mathrm{min}$ on peut observer les raies de l'azote $(415,146 \mathrm{~nm}$ $413,763 \mathrm{~nm}-410,998 \mathrm{~nm}$ ).

Intensité : 15 A et $30 \mathrm{~A}$.

3. Choix des raies. - Les critères de choix ne sont pas les mêmes pour les différents constituants du plasma :

Argon : les raies choisies ne sont pas ou très peu auto-absorbées et leurs écarts en énergie sont suffisants. Elles ne se superposent à aucune raie du cuivre. Nous avons retenu les raies suivantes :

$415,854 \mathrm{~nm} \quad 427,217 \mathrm{~nm} \quad 696,543 \mathrm{~nm} \quad 706,722 \mathrm{~nm}$.

Les valeurs de $E_{m}, A_{m n}, g_{m}$ sont données par [12].

Cuivre : on a choisi des raies pour lesquelles les probabilités de transition sont connues avec une précision correcte. Corliss [13] a fait une étude récapitulative des différents résultats concernant les probabilités de transition de certaines raies du cuivre. Conformément à sa conclusion nous avons retenu celles de Kock et Richter [14]; de plus les conditions expérimentales dans lesquelles cette détermination a été faite (arc stabilisé sous atmosphère d'argon) sont proches des nôtres. Il faut tenir compte également de l'auto-absorption. Pichler, Vujnovic, Tonejc et Acinger [3] justifient par des résultats expérimentaux ainsi que par des considérations théoriques que les raies 515 et $521 \mathrm{~nm}$ ne sont pas auto-absorbées. Les états $4 \mathrm{p}^{2}{ }^{4} \mathrm{~F}_{9 / 2}^{0}, 4 \mathrm{~s}^{2}{ }^{2} \mathrm{D}_{5 / 2}$ et $4 \mathrm{~s}^{2}{ }^{2} \mathrm{D}_{3 / 2}$ étant métastables, les-transitions $465,510,570$ et $578 \mathrm{~nm}$ sont suspectes d'auto-absorption. Miyachi, Kito et Okada [2] ont vérifié que la raie $510 \mathrm{~nm}$ (ainsi que la $521,8 \mathrm{~nm}$ ) n'est pas auto-absorbée. Les résultats obtenus à partir des raies 465,570 et $578 \mathrm{~nm}$ devront être interprétés avec prudence en fonction de ceux obtenus avec les autres raies. Le tableau I donne les principales caractéristiques des raies utilisées. La précision est donnée par Kock et Richter.

4. Résultats expérimentaux. - Différentes sortes de mesures ont été faites. Pour des régimes d'arc

Tableau I. - Caractéristiques des raies du cuivre.

[Characteristics of copper line spectra.]

\begin{tabular}{|c|c|c|}
\hline \multirow{2}{*}{$\begin{array}{l}\text { Longueur d'onde } \\
\text { (nm) }\end{array}$} & \multicolumn{2}{|c|}{ Transition } \\
\hline & niveau $n$ & niveau $m$ \\
\hline - & & - \\
\hline 465,1124 & $4 p^{2 ~}{ }^{4} F_{9 / 2}^{0}$ & $5 s^{\prime}{ }^{4} D_{7 / 2}$ \\
\hline 510,5541 & $4 s^{2}{ }^{2} D_{5 / 2}$ & $4 p^{2} \quad P_{3 / 2}^{0^{1}}$ \\
\hline 515,3235 & $4 p^{2} \quad P_{1 / 2}^{0}$ & $4 d^{2} \quad D_{3 / 2}$ \\
\hline 521,8202 & $4 p^{2} P_{3 / 2}^{0}$ & $4 d^{2} \quad D_{5 / 2}$ \\
\hline 570,0240 & $4 s^{2}{ }^{2} D_{3 / 2}$ & $4 p^{2} \quad P_{3 / 2}^{0}$ \\
\hline 578,2132 & $4 s^{2}{ }^{2} D_{3 / 2}$ & $4 p^{2} \quad P_{1 / 2}^{0}$ \\
\hline
\end{tabular}

$\begin{array}{ccc}\begin{array}{c}E_{m} \\ (\mathrm{eV})\end{array} & \log (g f) & g_{m} \\ -\overline{-} & - & - \\ 7,74 & -0,01 & 8 \\ 3,82 & -1,51 & 4 \\ 6,19 & -0,01 & 4 \\ 6,19 & +0,27 & 6 \\ 3,82 & -2,34 & 4 \\ 3,79 & -1,78 & 2\end{array}$

$\begin{gathered}A_{m n} \\ \left(\mathrm{~s}^{-1}\right)\end{gathered}$
-
$3,80 \times 10^{7}$
$0,20 \times 10^{7}$
$6,04 \times 10^{7}$
$7,50 \times 10^{7}$
$0,024 \times 10^{7}$
$0,165 \times 10^{7}$

Précision (K.R.)

$12 \%$

$12 \%$

$12 \%$

$12 \%$

$14 \%$

$12 \%$ 
exempt de contamination métallique obtenus dans une chambre à ouverture latérale, nous avons :

- comparé les valeurs de la température sur l'axe avec celles obtenues dans une chambre de Maecker,

- tracé les isothermes de la colonne afin de pouvoir les comparer à celles obtenues lorsque le plasma est contaminé par des valeurs anodiques de cuivre.

Dans un plasma contaminé nous avons ensuite déterminé les répartitions radiales de la température, la densité électronique, la teneur en cuivre dans des sections droites situées à différentes distances de l'anode.

4.1 Plasma D'ARgon eXempt de VaPeurs métalliQUES. - Dans le cas de la chambre à ouverture latérale la symétrie de révolution, indispensable pour appliquer l'inversion de l'équation intégrale d'Abel, est difficile à vérifier mais on peut penser qu'elle ne sera pas affectée car on constate que la colonne d'arc, beaucoup plus fine avec les électrodes $\mathrm{W}^{-}$et $\mathrm{Cu}^{+}$ (ou $\mathrm{Cu}-\mathrm{Zr}^{+}$) qu'avec des électrodes au carbone, est essentiellement stabilisée par le vortex gazeux. Un des rôles des parois de la chambre est (outre le refroidissement) la création et le maintien de ce vortex d'argon.

Pour certains débits d'argon, avec les électrodes métalliques $\left(\mathrm{W}^{-} \mathrm{Cu}^{+}\right.$ou $\left.(\mathrm{CuZr})^{+}\right)$nous avons obtenu des régimes d'arc qui peuvent être considérés comme exempts de vapeurs métalliques dans les zones explorées. Nous avons effectué des mesures de température à $15,30,40 \mathrm{~A}$. La figure 2 représente les températures obtenues sur l'axe de la décharge pour les 2 types de chambre. La dispersion faible à $15 \mathrm{~A}$ et $30 \mathrm{~A}$ (respectivement $1 \%$ et $3 \%$ ) semble plus importante à $40 \mathrm{~A}$ où elle atteint $7 \%$.

Nous pouvons néanmoins considérer que pour $15 \mathrm{~A}$ et $30 \mathrm{~A}$, intensités pour lesquelles nous ferons des mesures en présence de vapeurs métalliques, la concordance est satisfaisante.

Une éventuelle imprécision due à l'ouverture latérale de la chambre sera d'ailleurs toujours très inférieure à celle des résultats obtenus dans les plasmas contaminés par des vapeurs métalliques.

A 30 A pour des points de visée situés à 2, 5, 8, 11, 14

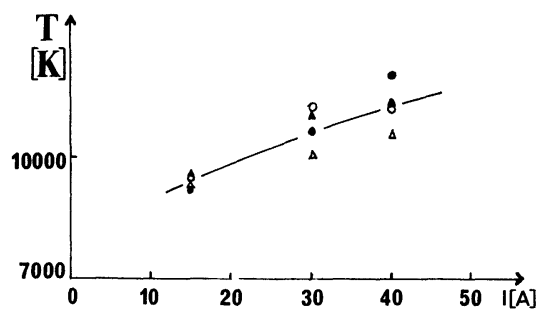

Fig. 2. - Température sur l'axe déterminée par intensité relative $(O \Delta)$ et par intensité absolue $(\Delta \Delta)$ de raies pour une chambre de Maecker $(\mathrm{O} \bullet)$ et une chambre à ouverture latérale $(\Delta \boldsymbol{\Delta})$.

[Temperature on the axis determined by relative and absolute intensities $(O \Delta),(\boldsymbol{\Delta})$ of line spectra for a Maecker chamber $(\mathrm{O} \bullet)$ and a side-slit chamber $(\Delta \Delta)$.] et $17 \mathrm{~mm}$ de l'anode, nous avons tracé les isothermes (Fig. 3) qui sembleraient mettre en évidence une légère augmentation de température au voisinage de l'anode.

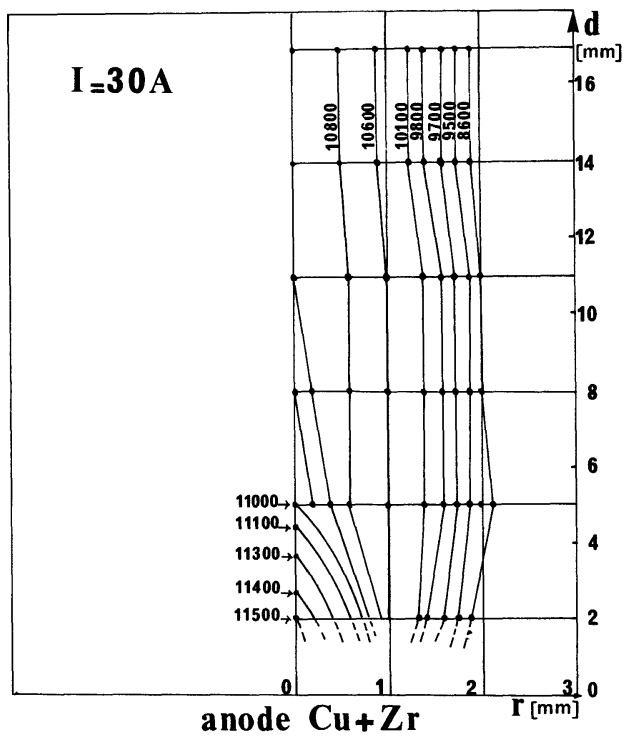

Fig. 3. - Isothermes de la colonne d'argon sans vapeurs métalliques $\left(\mathrm{W}^{-} \mathrm{Cu}^{+}-I=30 \mathrm{~A}\right)$.

[Isotherms of argon column, without metal vapour

$$
\left.\left(\mathrm{W}^{-} \mathrm{Cu}^{+}-I=30 \mathrm{~A}\right) \cdot\right]
$$

4.2 Plasma d'argon CONTAMiné Par des Vapeurs ANODIQUES DE CUIVRE. - Les mesures dans ce type de plasma se sont révélées très délicates en raison de l'instabilité dans le temps de l'émission anodique. En cours de mesure il peut se produire :

- une émission brutale de gouttelettes de métal, - un jet latéral de vapeur métallique qui détruit la symétrie de révolution,

- une décroissance lente dans le temps de l'intensité des raies du cuivre due à une usure trop rapide de l'anode dont la cause est un refroidissement légèrement insuffisant (il est important de placer l'anode de façon à ce qu'elle dépasse à peine du porteélectrode de refoidissement).

Un très grand nombre de mesures ont permis d'en dégager plusieurs séries pour lesquelles les conditions expérimentales sont satisfaisantes et ont, en outre, permis de montrer que les résultats obtenus sur l'intensité absolue des deux raies de l'argon (415,8 et $696,5 \mathrm{~nm}$ ) étaient toujours identiques à ceux obtenus sur les 4 raies citées au paragraphe 3 . Les résultats expérimentaux donnés par la suite ne concernent que ces 2 raies (temps de mesure moins long). La figure 4 montre les courbes de répartition radiale de la température pour un arc à $30 \mathrm{~A}$, visée à $1 \mathrm{~mm}$ et à $4 \mathrm{~mm}$ de l'anode. Chaque couple de valeurs d'intensité totale de raie (1 raie du cuivre, 1 raie de l'argon) permet de déterminer une valeur de $T, X$ et $N_{\mathrm{e}}$. Les 12 combinaisons raie Ar-raie $\mathrm{Cu}$ donnent des résultats concordants. 

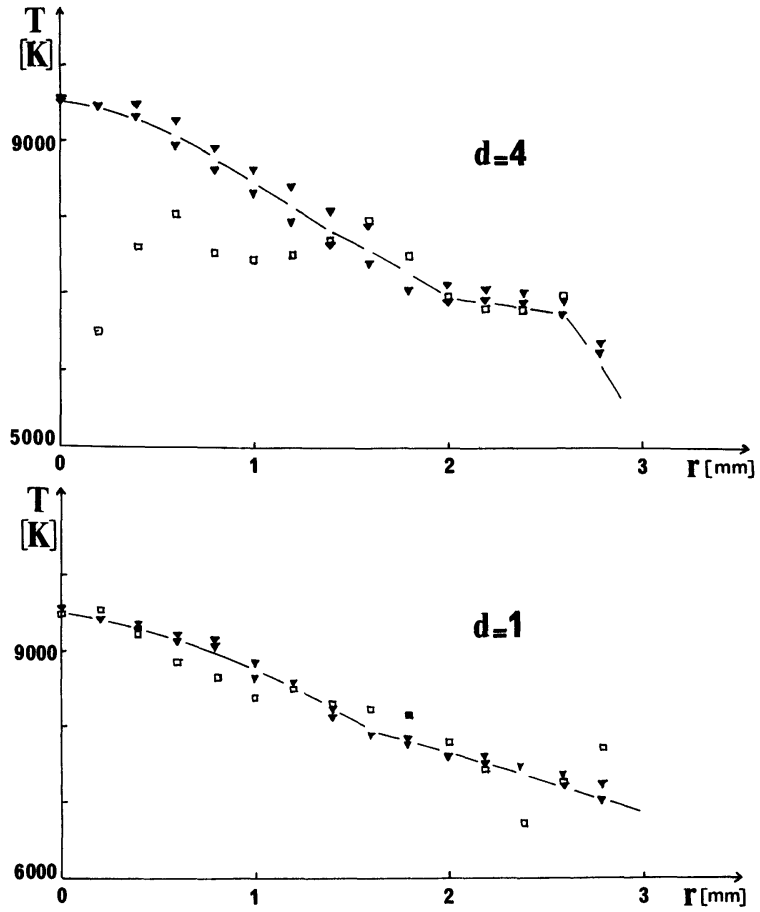

Fig. 4. - Répartitions radiales de température à $1 \mathrm{~mm}$ et $4 \mathrm{~mm}$ de l'anode $(I=30 \mathrm{~A})$ obtenues par la méthode des intensités absolues de raies $(\mathbf{\Lambda})$ et par celle des intensités relatives sur les 6 raies du

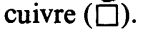

[Radial temperature distribution at $1 \mathrm{~mm}$ and $4 \mathrm{~mm}$ from anode $(I=30 \mathrm{~A})$ determined by absolute intensities of line spectra $(\Delta)$ and by relative intensities of six copper line spectra $(\square)$.]

Quand on s'écarte de l'anode les courbes de répartition radiale d'intensité des raies du cuivre changent de forme et présentent un léger creux au centre comme le montre la figure 5 . Le calcul du coefficient d'émissivité locale par inversion de l'équation intégrale d'Abel devient alors très délicat (une fluctuation de $2 \%$ au creux de la courbe expérimentale entraîne une variation de $18 \%$ sur le coefficient d'émissivité locale). Il ne sera pas possible de faire une détermination précise de $X$ au centre de la décharge lorsqu'on s'éloigne de l'anode $(4,8 \mathrm{~mm})$. Les températures obtenues

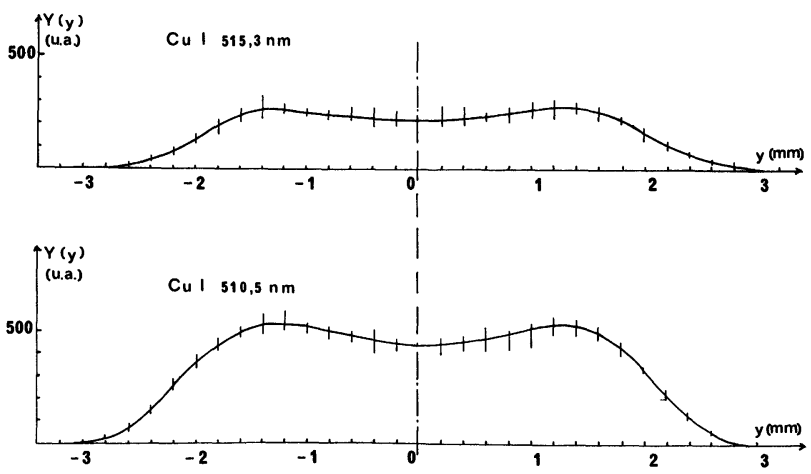

Fig. 5. - Répartitions radiales des raies du cuivre $(515,3 \mathrm{~nm}$ et $510,5 \mathrm{~nm})$.

[Radial distribution of copper line spectra $(515.3 \mathrm{~nm}$ to $510.5 \mathrm{~nm})$.]

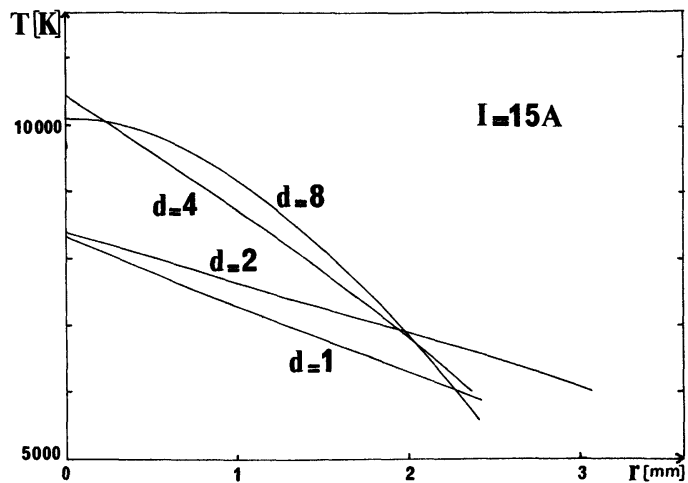

Fig. 6. - Répartition radiale de température $(I=15 \mathrm{~A}, d=1,2$, $4,8 \mathrm{~mm}$ ).

[Radial distribution of temperature $(I=15 \mathrm{~A}, d=1,2,4,8 \mathrm{~mm})$.]

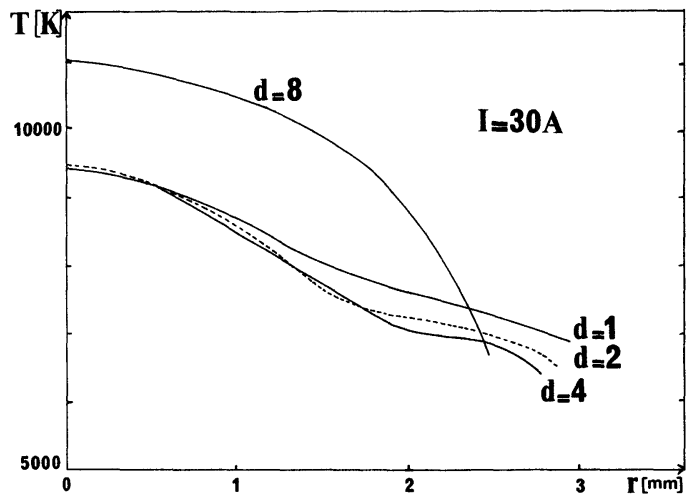

Fig. 7. - Répartition radiale de température $(I=30 \mathrm{~A}, d=1,2$, 4, $8 \mathrm{~mm}$ ).

[Radial distribution of temperature $(I=30 \mathrm{~A}, d=1,2,4,8 \mathrm{~mm})$.]

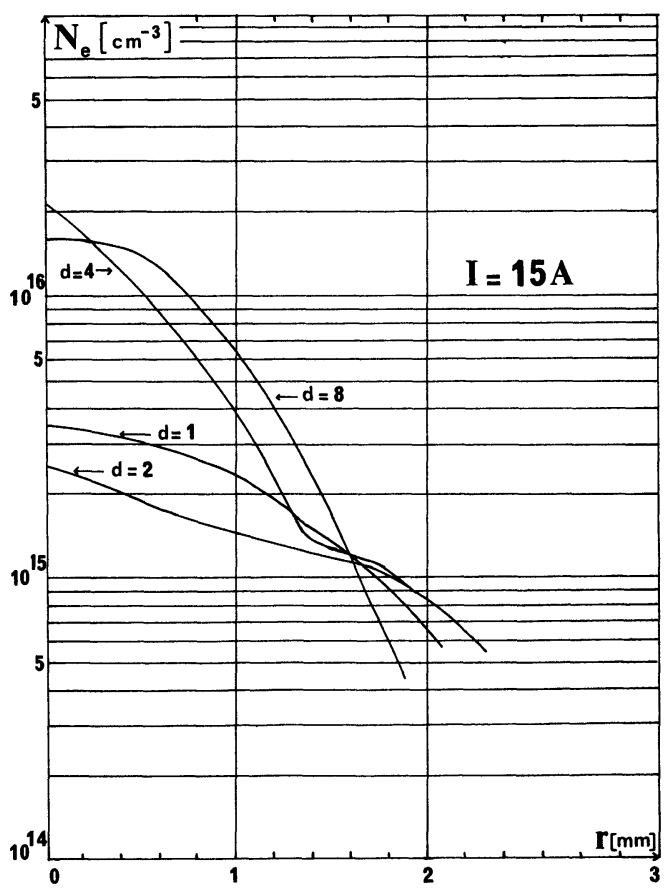

Fig. 8. - Répartition radiale de densité électronique $N_{\mathrm{e}}(I=15 \mathrm{~A}$; $d=1,2,4,8 \mathrm{~mm}$ ).

[Radial distribution of electron density $N_{\mathrm{e}}(I=15 \mathrm{~A}, d=1,2$, $4,8 \mathrm{~mm})$. 
par intensité relative des raies du cuivre seront pour la même raison en mauvais accord avec les autres résultats au centre de la décharge lorsque $d \geqslant 4 \mathrm{~mm}$. La détermination de $T$ par la méthode d'intensité absolue, qui dépend essentiellement des raies de

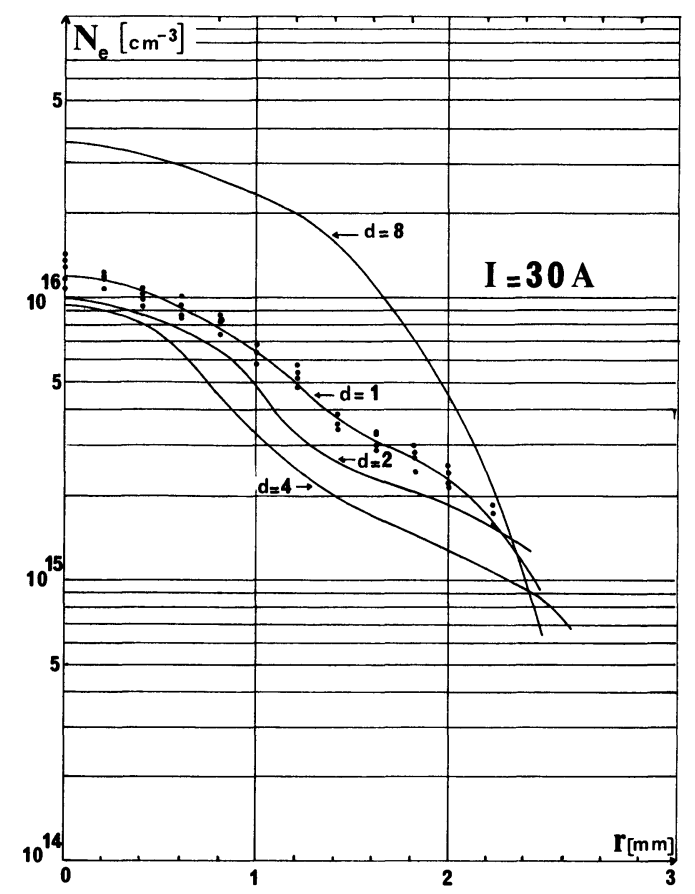

Fig. 9. - Répartition radiale de densité électronique $N_{\mathrm{e}}(I=30 \mathrm{~A}$; $d=1,2,4,8 \mathrm{~mm}$ ).

[Radial distribution of electron density $N_{\mathrm{e}}(I=30 \mathrm{~A}, d=1,2$, $4,8 \mathrm{~mm})$.]

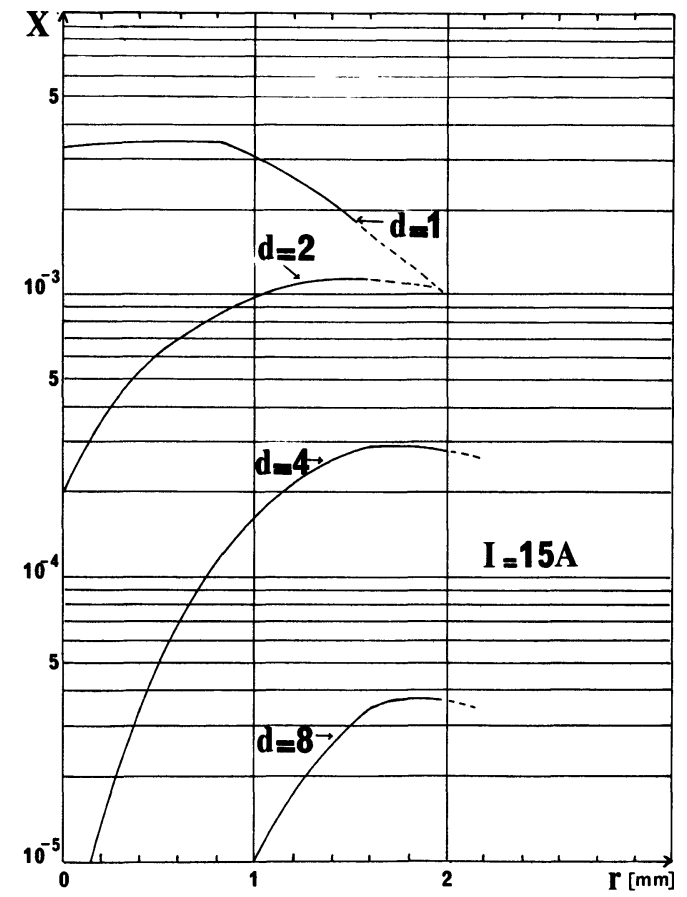

Fig. 10. - Répartition radiale de la teneur en cuivre $X(I=15 \mathrm{~A}$; $d=1,2,4,8 \mathrm{~mm}$ ).

[Radial distribution of copper content $X(I=15 \mathrm{~A}, d=1,2$, $4,8 \mathrm{~mm})$.] l'argon, sera au contraire plus précise au centre que sur les bords.

Les résultats figurant sur la figure 4 permettent d'apprécier la dispersion des mesures. La température est déterminée avec une précision de l'ordre de $300 \mathrm{~K}$. Les figures 9 et 11 donnent les valeurs de $N_{\mathrm{e}}$ et de $X$ pour une section droite de la décharge située à $1 \mathrm{~mm}$ de l'anode; l'intensité dans l'arc étant de $30 \mathrm{~A}$. La précision sur $N_{\mathrm{e}}$ est de l'ordre de $30 \%$, sur $X$ elle est faible et peut être de l'ordre de $100 \%$. Ces mesures permettent néanmoins d'obtenir un ordre de grandeur de la teneur de cuivre dans la décharge. Les figures 6 , $7,8,9,10,11$ donnent les résultats obtenus à $15 \mathrm{~A}$ et 30 A pour $T, X$ et $N_{\mathrm{e}}$ pour 4 distances de l'anode.

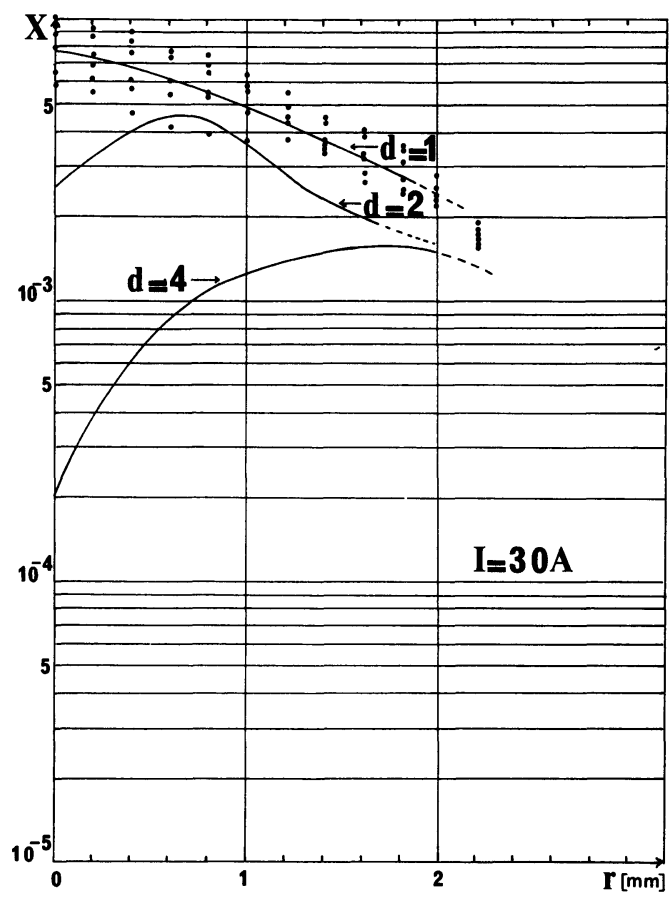

Fig. 11. - Répartition radiale de la teneur en cuivre $X(I=30 \mathrm{~A}$; $d=1,2,4,8 \mathrm{~mm}$ ).

[Radial distribution of copper content $X(I=30 \mathrm{~A}, d=1,2,4$, $8 \mathrm{~mm})$.]

5. Analyse des résultats. - De façon à mettre davantage en évidence la répartition du cuivre dans la colonne, nous avons tracé (Fig. 12) les cartes d'isodensité à $15 \mathrm{~A}$ et $30 \mathrm{~A}$ qui montrent de manière très nette une concentration importante du cuivre à la périphérie. Ce phénomène pourrait être dû à la diffusion ambipolaire des impuretés qui, lorsqu'elles ont un potentiel d'ionisation inférieur à celui du gaz porteur, s'ionisent entre l'axe et le bord et diffusent ensuite vers les parois [15].

La précision très médiocre obtenue dans la détermination de $X$ ne remet pas en question la forme générale des courbes puisque ce paramètre varie dans un rapport de 1 à 100 . La différence de précision dans la détermination de $T$ et celle de $X$ peut s'expli- 

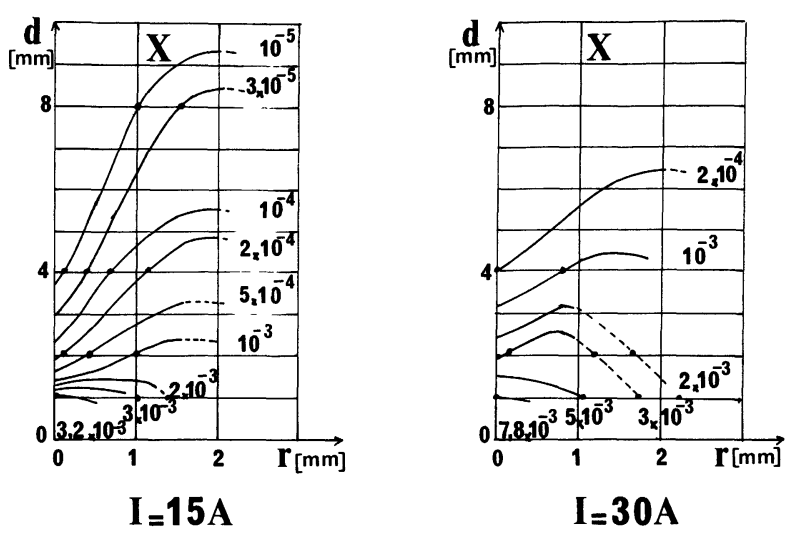

Fig. 12. - Variation de la teneur en cuivre dans la colonne $(I=15 \mathrm{~A}$ et $30 \mathrm{~A}$ ).

[Variation of copper content in the column $(I=15 \mathrm{~A}$ and $30 \mathrm{~A})$.]

quer à partir du calcul théorique exposé dans [8]. Nous avons démontré que la détermination de la température dépend essentiellement de l'intensité absolue des raies de l'argon qui varie très peu en fonction de $X$ tant que celui-ci est inférieur à 1 , ce qui sera toujours le cas expérimentalement. La mesure de $T$ sera donc très peu affectée par les fluctuations de l'émission anodique, les paramètres $X$ et $N_{\mathrm{e}}$ sont par contre déterminés à partir de l'intensité absolue des raies du cuivre et de l'argon et seront de ce fait très liés aux fluctuations expérimentales.

Les figures 8 et 9 mettent en évidence l'apport électronique du cuivre dans les zones périphériques de la décharge $(d=1,2,4 \mathrm{~mm})$. On peut tracer, pour confirmer ce fait, les courbes donnant le pourcentage d'électrons venant du cuivre en différents

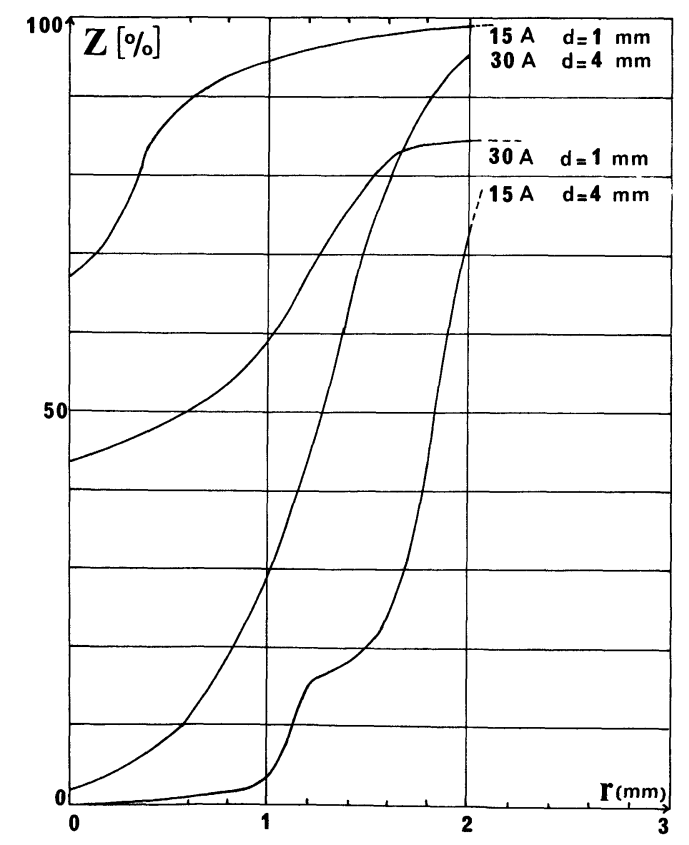

Fig. 13. - Variation du pourcentage $Z$ d'électrons dus au cuivre. [Variation of percentage of electrons $Z$ due to copper.] points de la colonne (Fig. 13). Ces valeurs ont été obtenues, comme celles de $T, X, N_{\mathrm{e}}$, par résolution du système d'équations du paragraphe 1 . La forme des courbes est aisément expliquable étant donné le faible potentiel d'ionisation du cuivre par rapport à celui de l'argon.

En comparant les isothermes de la figure 2 à celles de la figure 14 , nous pouvons constater un abaissement de température important au voisinage de l'anode (de l'ordre de $2000 \mathrm{~K}$ ) quand il y a émission de cuivre. C'est l'introduction dans la colonne d'éléments à faible potentiel d'ionisation (même à faible concentration) qui d'après [16] produit cet abaissement relativement important.
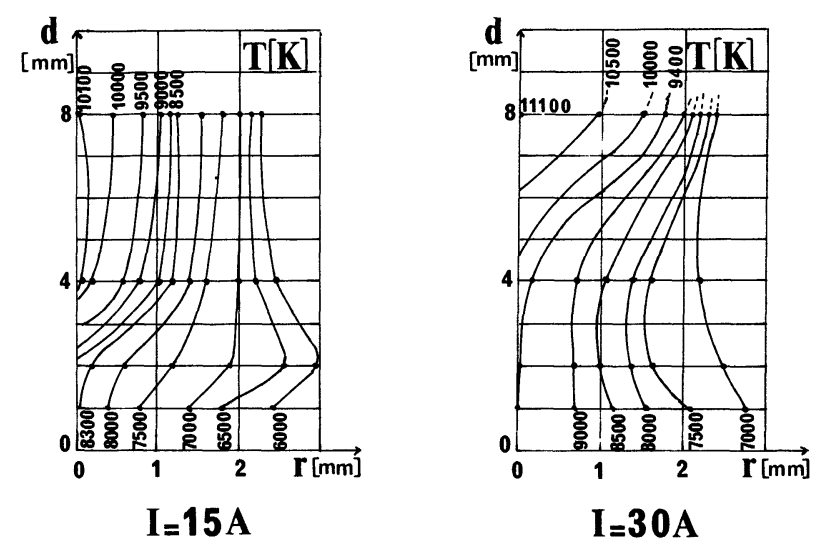

Fig. 14. - Isothermes de la colonne (en présence de vapeurs métalliques).

[Column isotherms (in the presence of metal vapour).]

Le calcul de $T, X$ et $N_{\mathrm{e}}$ à partir du résultat des mesures suppose l'équilibre thermodynamique. Cette méthode utilisée pour les 6 raies de cuivre citées conduit à des valeurs de $T, X$ et $N_{\mathrm{e}}$ relativement bien concordantes. Il est bien évident que l'ensemble de ces résultats n'est pas suffisant pour affirmer que l'hypothèse de l'E.T.L. est vérifiée d'autant plus que 3 des 6 raies du cuivre utilisées ne sont peut-être pas exemptes d'auto-absorption.

La comparaison des valeurs de $N_{\mathrm{e}}$ avec celles déterminées par une autre méthode serait intéressante. Dans ce but nous avons envisagé :

- La mesure de l'élargissement par effet stark des raies du cuivre [17] ou de l'argon [18]. Dans les 2 cas les élargissements (de l'ordre de 0,001 nm) sont trop faibles pour être mesurés avec l'appareillage dont nous disposons.

- La mesure de la largeur à mi-hauteur de la raie $\mathrm{H}_{\beta}$. Nous avons introduit $1 \%$ d'hydrogène dans l'argon, on peut distinguer 2 zones. Dans la première les raies du cuivre sont très faibles ou nulles et la mesure de la largeur à mi-hauteur de la raie $\mathrm{H}_{\beta}$ permet de déterminer des valeurs de $N_{\mathrm{e}}$ qui sont à $20 \%$ près celles que l'on peut déterminer dans un plasma d'argon pur. Dans la deuxième zone, les raies du cuivre sont 
mesurables, mais alors celles de l'argon sont faibles et on ne peut pas tracer le profil de la raie $\mathrm{H}_{\beta}$. L'introduction de $1 \%$ d'hydrogène dans l'argon pouvant modifier notablement le plasma, il serait de toute manière difficile de faire des comparaisons significatives.

6. Conclusion. - Malgré des conditions expérimentales délicates, les mesures ont permis de déterminer l'ordre de grandeur des différents paramètres caractérisant ce plasma et de mettre en évidence un phénomène intéressant d'expulsion du cuivre vers la périphérie de la colonne.
Aucune comparaison n'a été possible avec d'autres résultats du fait qu'à notre connaissance, aucun travail sur la détermination des paramètres caractérisant un plasma d'argon contaminé par des vapeurs anodiques de cuivre n'a été publié jusqu'alors. En effet, les études citées dans l'introduction ont été réalisées dans des conditions expérimentales très différentes.

Si l'ensemble des résultats obtenus est cohérent, il est bien certain que leur exploitation a nécessité des hypothèses dont l'influence est pour l'instant difficile à apprécier. Aussi d'autres études expérimentales et théoriques seront encore nécessaires.

\section{Bibliographie}

[1] Norman, P., Fells, I., Trans. Instrum. Chem. Eng. 55 (1977) 149-152.

[2] MiYachi, I., Kito, Y., OKadA, K., J. Instrum. Elect. Eng. Japan 87 (1967) 1227-34.

[3] Pichler, G., Vujnovic, V., Tonejc, A. M., Acinger, K., Spectrochim. Acta 27B (1972) 273-286.

[4] Jayaram, K., Z. Phys. 271 (1974) 217-220.

[5] Miyachi, I., Jayaram, K., Asai, Y., Proc. 2nd Int. Conf. on gas Discharges. London (1972) 364-6.

[6] Airey, D. R., Kinsinger, R. E., Richards, P. H., Swift, J. D., I.E.E.E. Trans. Power apparatus and systems. PAS-95 (1976) 1-13.

[7] Andanson, P., Cheminat, B., Halbique, A. M., J. Phys. D 11 (1978) 209-215.

[8] Andanson, P., Cheminat, B., Composition d'équilibre d'un plasma argon-cuivre (à paraître)

[9] GrIEM, H. R., Plasma spectroscopy (McGraw-Hill Book Company New York) 1964.
[10] Drawin, H. W., Felenbok, P., Data for plasmas in local thermodynamic equilibrium (Gauthier-Villars Editeur, Paris) 1965.

[11] Andanson, P., Cheminat, B., Lefort, A., Roche, J., Rev. Gen. Electr. 88 (1979) (à paraître).

[12] Atomic transition Probabilities. Nat. Stand. Ref. Data Ser., Nat. Bur. Stand. 22 (1969) vol. II.

[13] Coruss, C. H., J. Res. Nat. Bur. Stand. 74A (1970) 781-790.

[14] Kock, M., RichteR, J., Z. Astrophys. 69 (1968) 180-192.

[15] Maecker, J., Discharge and plasma physics. Ecole d'été, Université de New England, Australie (Janvier 1963).

[16] Boumans, P. W. J. M., Theory of Spectrochemical excitation (Hilger \& Watts LTD, London), 1966.

[17] JAYARAM, K., Theoretical and experimental investigation of a copper vapour arc with special reference to the spectral line widths. Thèse 1971 .

[18] Preston, R. C., J. Quant. Spectrosc. Radiat. Transfer 18 (1977) 337-360. 